\title{
On Cosmic Rays, IP Structures and Geospace Consequences During WHI
}

\author{
A. Dal Lago ${ }^{1}$, F. L. Guarnieri ${ }^{2}$, M. R. da Silva ${ }^{1}$, W. D. Gonzalez ${ }^{1}$, \\ C. R. Braga ${ }^{1}$, N. J. Schuch ${ }^{3}$, K. Munakata ${ }^{4}$, C. Kato ${ }^{4}$, J. W. Bieber ${ }^{5}$, \\ T. Kuwabara ${ }^{5}$, M. Tokumaru ${ }^{6}$, M. L. Duldig ${ }^{7}$ and J. E. Humble ${ }^{8}$ \\ ${ }^{1}$ National Institute for Space Research (INPE), Sao Jose dos Campos, Brazil \\ email: dallago@dge.inpe.br \\ ${ }^{2}$ Universidade do Vale do Paraiba (UNIVAP), Sao Jose dos Campos, Brazil \\ ${ }^{3}$ Southern Regional Space Research Center (CRS/INPE), Santa Maria, Brazil \\ ${ }^{4}$ Physics Department, Shinshu University, Japan \\ ${ }^{5}$ Bartol Research Institute and Department of Physics and Astronomy, Univ. of Delaware, USA \\ ${ }^{6}$ Solar-Terrestrial Environment Laboratory, Nagoya University, Japan \\ ${ }^{7}$ Australian Antarctic Division, Tasmania, Australia \\ ${ }^{8}$ School of Mathematics and Physics, University of Tasmania, Tasmania, Australia
}

\begin{abstract}
This work presents some observations during the period of the Whole Heliosphere Interval (WHI) of the effects of interplanetary (IP) structures on the near-Earth space using three sets of observations: magnetic field and plasma from the Advanced Composition Explorer (ACE) satellite, ground-based cosmic ray data from the Global Muon Detection Network $($ GMDN) and geomagnetic indices (Disturbance storm-time, Dst, and auroral electrojet index, AE). Since WHI was near minimum solar activity, high speed streams and corotating interaction regions (CIRs) were the dominant structures observed in the interplanetary space surrounding Earth. Very pronounced geomagnetic effects are shown to be correlated to CIRs, especially because they can cause the so-called High-Intensity Long-Duration Continuous AE Activity (HILDCAAs) - Tsurutani and Gonzalez (1987). At least a few high speed streams can be identified during the period of WHI. The focus here is to characterize these IP structures and their geospace consequences.
\end{abstract}

Keywords. Cosmic Rays, CIRs, AE index, WHI

\section{Introduction}

In this work, we will address ground cosmic ray observations from the Global Muon Detector Network (GMDN) during the Whole Heliosphere Interval (WHI) period, CR2068, from March 20-April 16, 2008 (http://ihy2007.org/WHI/), as well as geomagnetic effects correlated to CIRs observed during this period.

\section{Global Muon Detection Network Observations During WHI}

Since December 1992, a two-hemisphere network has acquired data using a pair of muon detectors at Nagoya (Japan) and Hobart (Australia), which have detection areas of $36 \mathrm{~m}^{2}$ and $9 \mathrm{~m}^{2}$, respectively. A small $4 \mathrm{~m}^{2}$ prototype was installed in São Martinho da Serra (Brazil) in 2001 (Da Silva, et al. (2004)), which was upgraded in December 2005 , increasing its detection area to $28 \mathrm{~m}^{2}$ (Okazaki, et al. (2008)). These detectors are multidirectional, allowing the simultaneous recording of intensities in various viewing directions. In March 2006, the Global Muon Detector Network (GMDN) was completed 
with a new detector at Kuwait University (Kuwait), with a detection area of $9 \mathrm{~m}^{2}$. Unlike the others, this muon detector is a hodoscope designed specifically for measuring the loss cone anisotropy (Munakata, et al. (2000); Leerungnavarat, et al. (2003)).

Figure 1 shows observations from the GMDN for the first half of the WHI period, from DOY 85 to 90 . From top to bottom is shown the cosmic ray best-fit density, measured $\mathrm{x}, \mathrm{y}, \mathrm{z}$ anisotropies and calculated $\mathrm{x}, \mathrm{y}, \mathrm{z}$ cosmic ray gradient.

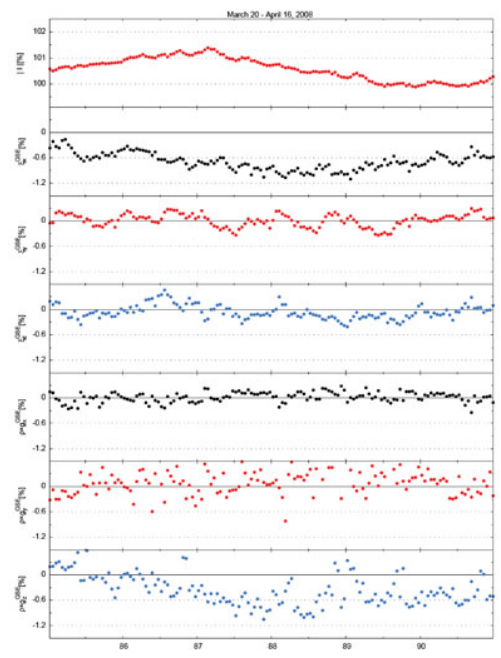

Figure 1. From top to bottom: cosmic ray best-fit density, measured $\mathrm{x}, \mathrm{y}, \mathrm{z}$ anisotropies and calculated $\mathrm{x}, \mathrm{y}, \mathrm{z}$ cosmic ray gradient.

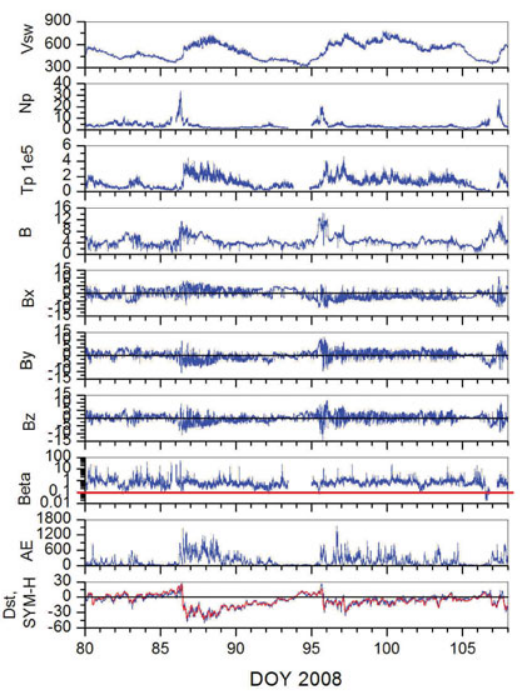

Figure 2. ACE solar wind velocity, proton number density and temperature, ACE IMF intensity, $x, y$ and $z$ components, plasma beta, auroral index (AE), Dst and SYM-H indeces.

From Figure 1, it is possible to see a negative $\mathrm{x}$ anisotropy during the period. Okazaki, et al. (2008), using GMDN observations, have interpreted similar anisotropies in terms of north-south cosmic ray gradients. In this particular case of Figure 1, a negative $\mathrm{x}$ anisotropy is consistent with the $A<0$ epoch of the solar cycle, for which a simple drift model predicts a local cosmic ray maximum in the Heliospheric Current Sheet (HCS) (see Okazaki, et al. (2008) for further details). ACE observations of IMF longitude (not shown) shows that the Earth was north of the HCS. Following the methodology of Okazaki, et al. (2008), the last panel of Figure 1 shows the cosmic ray gradient in the negative z direction, which is consistent with the fact that the Earth was north of the HCS.

\section{High Intensity, Long Duration, Continuous AE Activity Observations During WHI}

Tsurutani and Gonzalez (1987) defined a class of events which they called HILDCAAs, which stands for High Intensity, Long Duration, Continuous AE Activity. Its been debated that these events are not continuous substorms, but a new form of energy deposition in the auroral ionosphere (Tsurutani, et al. (2004); Guarnieri (2006)). The criteria for an event to be called HILDCAAs are: (1) AE peak of, at least, $1000 \mathrm{nT}$; (2) the event must last for, at least, two days; (3) the AE values cannot decrease to less than $200 \mathrm{nT}$ for 
more than 2 hours at a time; and (4) the event must occur outside the main phase of a geomagnetic storm. The interplanetary magnetic field during these events presents large amplitude fluctuations (Alfvén waves) instead of southward directed fields (Tsurutani and Gonzalez (1987); Gonzalez, et al. (1999)). HILDCAAs are found to be important in the descending and minimum phase of the solar cycle (Guarnieri (2006)), such as the WHI period. Figure 2 shows interplanetary observations from the Advanced Composition Explorer (ACE satellite) and geomagnetic indices from the WHI period (CR 2068). From top to bottom is shown ACE/SWEPAM solar wind velocity, proton number density and temperature, ACE/MAG IMF intensity, $\mathrm{x}, \mathrm{y}$ and $\mathrm{z}$ components, plasma beta, auroral index (AE), Dst and SYM-H indeces.

Following the above mentioned criteria, two periods of HILDCAAs are clearly identified in Figure 2, from DOY 86 to 90 and from DOY 96 to 100. These two periods are in close correspondence with two CIRs observed during this period. The CIRs can be identified as the two periods in Figure 2 with high velocities (first panel), preceded by high density peaks (second panel). Highly fluctuating magnetic field components can be identified in the IMF components (fifth, sixth and seventh panels). The auroral electrojet, AE, index is intensified during these events while the disturbance storm-time, Dst, and SIM-H indices do not reach values below $-50 n T$, indicating weak geomagnetic storm activity.

\section{Summary}

In this work we presented observations from the GMDN from the WHI period, as well as the geospace consequences associated with the interplanetary structures present in the near-Earth space. Cosmic ray anisotropies and gradients derived from the GMDN data are consistent with $A<0$ model predictions, following the work done by Okazaki, et al. (2008). Two CIRs were identified during the WHI period using ACE observations. These structures caused HILDCAA events. Such events are more frequent during descending and minimum phases of the solar cycle, such as the WHI period.

\section{Acknowledgements}

The authors would like to acknowledge N. Ness (Bartol Research Institute), D. J. McComas (Southwest Research Institute), R. Lepping (NASA GSFC), K. Ogilvie (NASA GSFC), and the CDAWeb for ACE interplanetary magnetic field and plasma data; and Kyoto WDC for the Dst index, CNPq of Brazil for projects 303798/2008-4, 472031/ $2007-4$.

\section{References}

Da Silva, M. R., Contreira, D. B., Monteiro, S., Trivedi, N. B., Munakata, K., Kuwabara, T., \& Schuch, N. J. 2004, Astrophys. Space Sci. 290, 3-4

Gonzalez, W. D., Tsurutani, B. T., \& Clua de Gonzalez, A. L. 1999, Space Sci. Rev. 88

Guarnieri, F. L. 2006, In: Geophysical Monograph Series 167

Leerungnavarat, K., Ruffolo, D., Bieber, J. W. 2003, ApJ 593, 587

Munakata, K., J. W. Bieber, S. I. Yasue, C. Kato, M. Koyama, S. Akahane, K. Fujimoto,

Z. Fujii, J. E. Humble, \& M. L. Duldig 2000, J. Geophys. Res. 105, 27

Okazaki, Y., Fushishita, A., Narumi, T., Kato, C., Yasue, S., Kuwabara, T., Bieber, J. W., Evenson, P., Da Silva, M. R., Dal Lago, A., Schuch, N. J., Fujii, Z., Duldig, M. L., Humble, J. E., Sabbah, I., Kota, J., \& Munakata, K. 2008, ApJ 681, 1

Tsurutani, B. T. \& Gonzalez, W. D. 1987, Space Sci. Rev. 35

Tsurutani, B. T., W. D. Gonzalez, F. L. Guarnieri, Y. Kamide, X.-Y. Zhou, \& J. K. Arbal 2004, J. Atmosph. Sol. Terr. Phys. 66, 167 